\title{
Estimating the Sensitivity of IceCube to Signatures of Axion Production in a Galactic Supernova
}

\author{
The IceCube Collaboration ${ }^{\dagger}$ \\ $\dagger$ http://icecube.wisc.edu/collaboration/authors/icrc17_icecube \\ E-mail: sybenzvilpas.rochester.edu, rcrossapas.rochester.edu, \\ tnguy510u.rochester.edu
}

\begin{abstract}
We describe the sensitivity of the IceCube Neutrino Observatory to the creation of axions in a Type II core-collapse supernova (SN II) in the Galaxy. Axions are a light dark matter candidate, and their existence is well-motivated as a solution to the strong CP problem. In a supernova, axions behave much like neutrinos, efficiently removing energy from the explosion and cooling the system. As a result, neutrino production in an SN II is suppressed in the presence of axions, mainly affecting the seconds-long tail of the SN II neutrino light curve. The IceCube Observatory is sensitive to large numbers of $\mathrm{MeV}$ neutrinos from an SN II, which produce a collective rise in the hit rates of all photomultipliers in the detector. We present a shape analysis that can be used with IceCube data to discriminate the axion production scenario from standard SN II models.
\end{abstract}

Corresponding authors: Segev BenZvi ${ }^{1}$, Robert Cross ${ }^{* 1}$, Tri Nguyen ${ }^{1}$

${ }^{1}$ Department of Physics and Astronomy, University of Rochester, Rochester, NY, USA

35th International Cosmic Ray Conference - ICRC2017

10-20 July, 2017

Bexco, Busan, Korea

* Speaker. 


\section{Introduction}

Axions and axion-like particles (ALPs) are very light extensions to the Standard Model [1, 2, 3]. They were first hypothesized in 1977 as a consequence of the breaking of the Peccei-Quinn symmetry [4], which eliminates finely-tuned CP violating terms in the strong interaction. The Peccei-Quinn axion is a very light $\left(m_{a} \ll 1 \mathrm{eV}\right)$ pion-like pseudoscalar particle. It couples to Standard Model particles $i$ with a strength $g_{a i}$ proportional to its mass $m_{a}$. ALPs have properties similar to axions but $m_{a}$ and $g_{a i}$ can be independent.

Axions and ALPs may comprise a significant portion of the cold dark matter in the Universe, and a number of laboratory and astrophysical measurements are underway to study their properties. Most of these efforts focus on the coupling of axions and ALPs to photons. In the presence of an external magnetic field, ALPs can oscillate into photons and vice-versa [5]. In the laboratory, direct searches for axion-photon mixing are carried out using the LSW technique ("light shining through a wall"), in which sub-mm photons are transported through an opaque barrier by converting them to ALPs in a strong dipole magnet, and then converting them back to photons in a second magnet on the far side of the barrier. Indirect versions of the LSW measurement can be attempted by searching for photon-ALP oscillations in astrophysical sources with natural magnetic fields; for example, measurements of the conversion of axions from the Sun to keV photons [6], or measurements of anomalously hard TeV $\gamma$-ray spectra from distant AGN [7]. In the latter case the LSW "magnets" are the $B$ fields of the AGN and Milky Way, and the "barrier" is the infrared extragalactic photon field which attenuates $\mathrm{TeV} \gamma$ rays from cosmologically distant sources. Indirect measurements of ALP-photon mixing are also possible in searches for oscillation-induced irregularities in the GeV and $\mathrm{TeV}$ spectra of gamma-ray sources $[8,9,10]$.

Astrophysically, axions and ALPs are expected to behave like neutrinos, efficiently removing energy from stars and altering their evolution [11, 12, 13]. These effects carry over to highluminosity astrophysical explosions such as Type II core-collapse supernovae (SNe II). With respect to standard astrophysical models, the production of axions in SNe II will create a significant excess of gamma rays $[14,15,16]$ due to ALP-photon oscillations, as well as a significant deficit of neutrinos due to axion cooling (see e.g. [17] and [18]). The extreme luminosity of SNe II means that "gamma appearance" and "neutrino disappearance" measurements in a Galactic supernova can be used to achieve order-of-magnitude improvements in limits on the mass and coupling strength of axions and ALPs $[16,19]$. Observations of a neutrino deficit are of particular interest because they are uniquely sensitive to the coupling of axions to nucleons $[18,20]$.

In this proceeding we describe the sensitivity of the IceCube Neutrino Observatory to the deficit of neutrinos in a Galactic SN II due to the effects of axion-nucleon coupling. We describe the IceCube detector and its sensitivity to supernovae in Section 2. The analysis technique and supernova model used are described in Section 3, followed by conclusions in Section 4.

\section{Supernova Detection with IceCube}

IceCube is a cubic kilometer Cherenkov detector located between $1.4 \mathrm{~km}$ and $2.4 \mathrm{~km}$ in the deep Antarctic ice below the South Pole. Using its array of 5160 digital optical modules (DOMs), 
the detector is triggered by the Cherenkov light produced when neutrinos $\gtrsim 100 \mathrm{GeV}$ interact in or near the detector.

The typical energy of neutrinos from a core-collapse supernova is $\sim 10 \mathrm{MeV}$, well below the trigger threshold of the main data acquisition system. The dominant interaction for supernova detection is $\bar{v}_{e}+p \rightarrow e^{+}+n$. A $10 \mathrm{MeV} e^{+}$, which is well above the Cherenkov threshold, loses its energy after traveling about $5 \mathrm{~cm}$ in the ice. Accounting for the Cherenkov emission of each positron, the efficiency of the DOMs, and the optical properties of the ice, a Galactic supernova will raise the count rate of each DOM by $2 \mathrm{~Hz}$ to $10 \mathrm{~Hz}$ for roughly 10 seconds, depending on the mass of the progenitor and its distance to Earth [21]. The average background count rate in the DOMs is about $280 \mathrm{~Hz}$ [21], and so the increase in the counts is not statistically significant at the level of individual channels. However, the correlated rise in the average background rate across the detector is highly statistically significant, with signal/background ratios $\gtrsim 10$ for an SN II located in the Milky Way [21].

In IceCube a dedicated supernova data acquisition system (SNDAQ) is used to search for neutrinos from a Galactic SN II in real time. SNDAQ monitors the background level in each DOM using a sliding 10-minute time window. In a central search window of width $0.5 \mathrm{~s}$ to $10 \mathrm{~s}$, it maximizes the likelihood of a collective rate deviation $\Delta \mu$ across all DOMs,

$$
\ln \mathscr{L}(\Delta \mu)=-\frac{1}{2}\left[\sum_{i=1}^{N_{\text {DOM }}}\left(\frac{r_{i}-\left(\left\langle r_{i}\right\rangle+\varepsilon_{i} \Delta \mu\right)}{\left\langle\sigma_{i}\right\rangle}\right)^{2}+\ln \left\langle\sigma_{i}\right\rangle+\ln 2 \pi\right],
$$

where $r_{i}$ is the hit rate of DOM $i$ in the search window, $\left\langle r_{i}\right\rangle$ and $\left\langle\sigma_{i}\right\rangle$ are the mean and RMS uncertainty in the background rate of DOM $i$, and $\varepsilon_{i}$ is the DOM efficiency. The maximum likelihood estimator $\Delta \hat{\mu}$ and its uncertainty $\sigma_{\Delta \hat{\mu}}$ are used to calculate the statistical significance $\xi=\Delta \hat{\mu} / \sigma_{\Delta \hat{\mu}}$. Statistically significant increases in $\Delta \mu$ produce real-time alerts for follow-up observations via the Supernova Early Warning System (SNEWS) [22].

\section{Analysis}

While SNDAQ produces alerts automatically and operates with $>99 \%$ uptime, the IceCube detector has not observed a Galactic supernova, and in fact no core-collapse supernovae have been observed in the Milky Way for hundreds of years. Therefore we perform an analysis of simulated data using the SN II neutrino light curve published by Fischer et al. [18], which compares an identical explosion with and without axion production in the proto-neutron star. In the following sections we describe the models and the shape analysis used to discriminate the two scenarios.

\subsection{Supernova Reference and Axion Models}

The process relevant for $\mathrm{SNe}$ II is nucleon-nucleon axion bremsstrahlung, described by the scattering $N_{1}+N_{2} \rightarrow N_{3}+N_{4}+a$. The phenomenological models used to describe axion-nucleon coupling are described in detail in [18], which assumes a Peccei-Quinn axion rather than an ALP. In brief, the coupling strength and axion mass scale like

$$
g_{a i}=C_{i} \frac{m_{N}}{f_{a}} \approx 10^{-7}\left(\frac{m_{a}}{\mathrm{eV}}\right) C_{i}, \quad m_{a} \approx 0.6 \mathrm{eV}\left(\frac{10^{7} \mathrm{GeV}}{f_{a}}\right),
$$


where $f_{a}$ is the Peccei-Quinn energy scale, $m_{N}$ is the nucleon mass, and $C_{i} \approx \mathscr{O}(0.1)$ is a constant that depends on the details of the hadronic axion model. Bounds on the axion-proton coupling from SN1987A yield

$$
g_{a p} \lesssim 9 \times 10^{-10}, \quad m_{a} \lesssim 3 \times 10^{-2} \mathrm{eV}, \quad f_{a} \gtrsim 5 \times 10^{8} \mathrm{GeV}
$$

for the coupling strength and mass of the PQ axion [18].
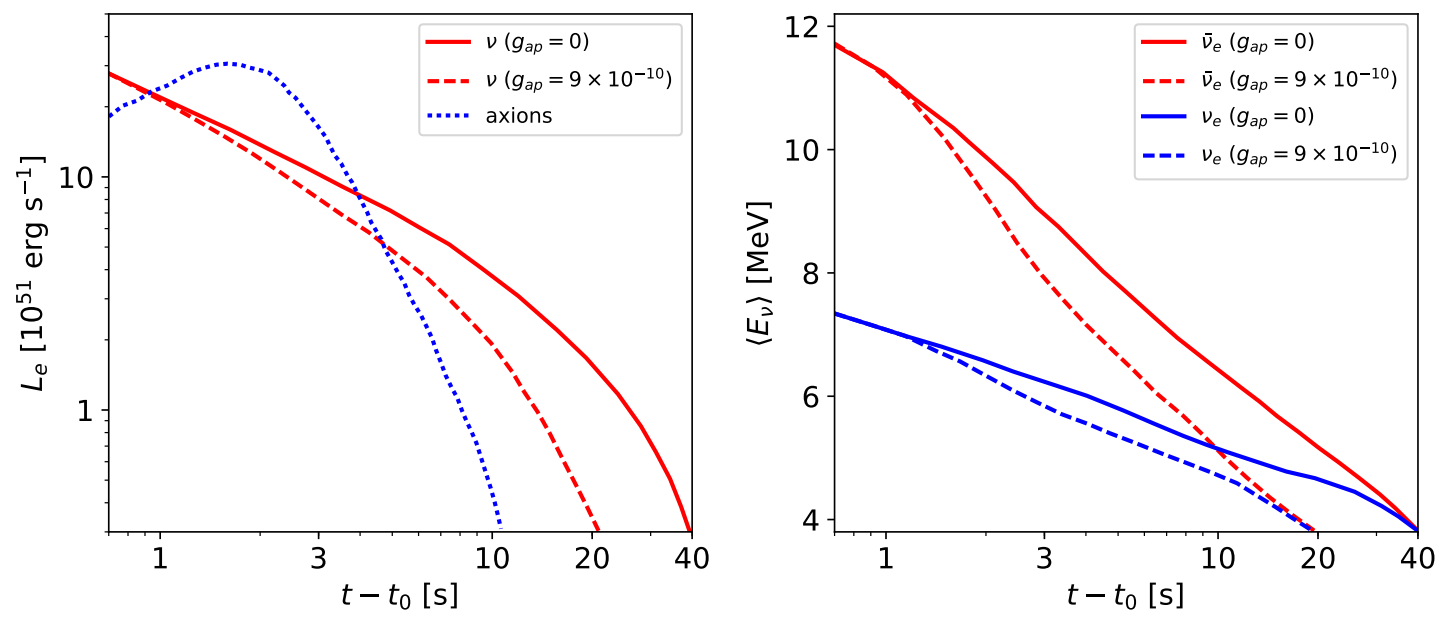

Figure 1: Neutrino and axion energy luminosity (left) and average neutrino energy for an SN II with an $18 M_{\odot}$ progenitor, as a function of time after core bounce $t_{0}$ (from [18]). At $t-t_{0}=10 \mathrm{~s}$, the nonzero axion-nucleon coupling reduces the neutrino luminosity by a factor of 2 and the average energy by $30 \%$ with respect to the reference model.
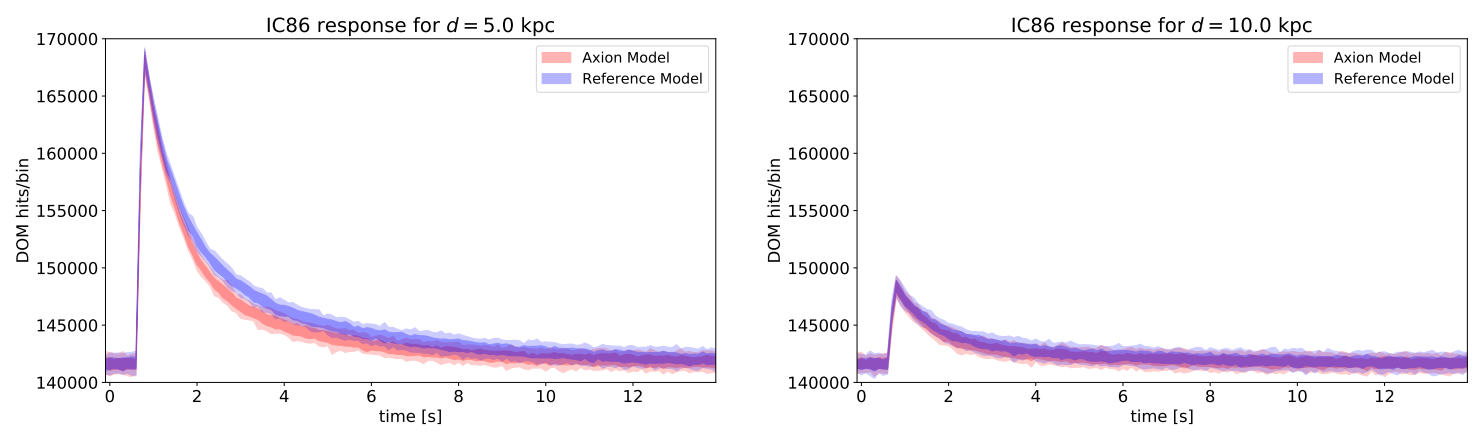

Figure 2: Simulated DOM hits in the completed 86-string configuration of IceCube (IC86) for a Galactic SN II located $5 \mathrm{kpc}$ from Earth (left) and $10 \mathrm{kpc}$ from Earth (right). A simulation using an $18 M_{\odot}$ progenitor from [18] was used to generate the DOM hits. The contours indicate the central $68 \%$ and $95 \%$ regions for 200 realizations of an explosion with axion production ("Axions") and without ("Reference").

Figure 1 shows the effect of axion production on the luminosity and average energy of neutrinos in an SN II from an $18 M_{\odot}$ progenitor [18]. An axion-proton coupling strength of $g_{a p}=$ $9 \times 10^{-10}$ has been assumed. While the neutrino luminosity at the breakout burst is not strongly affected by axion production, axions substantially reduce the length of the supernova cooling phase. 
Ten seconds after the core bounce, the neutrino luminosity is reduced by a factor of two compared to the reference model without axion production.

Using the neutrino light curves and energy distributions shown in Figure 1, we have simulated the response of the full IceCube detector to an $18 M_{\odot}$ SN II at a range of distances $d$ from Earth. In Fig. 2 the summed DOM hits are plotted as a function of time after the explosion for $d=5 \mathrm{kpc}$ and $d=10 \mathrm{kpc}$. The peak rate is unchanged if $g_{a p} \neq 0$, but the DOM hits in the cooling tail exhibit a clear decrease with respect to the reference model $\left(g_{a p}=0\right)$ before the rates return to the background level.

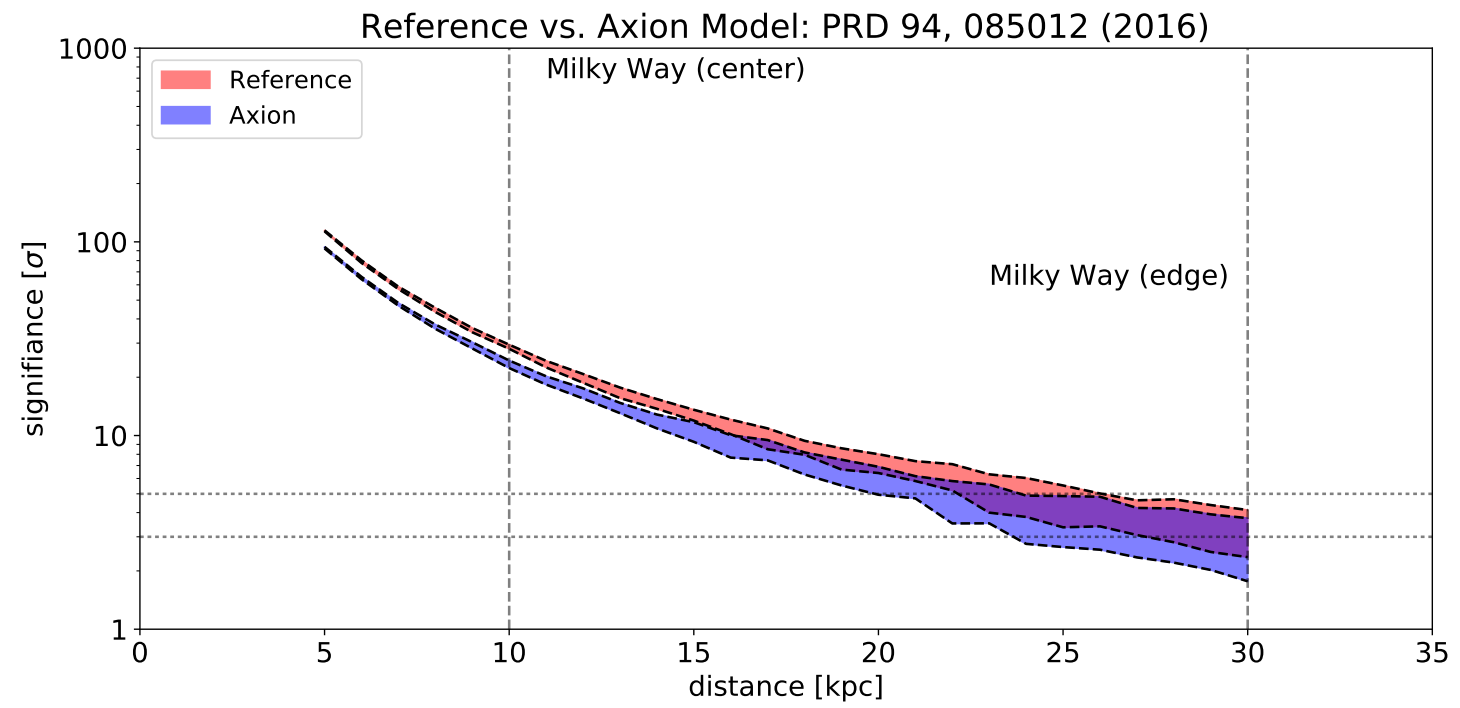

Figure 3: Sensitivity of IC86 to a Galactic SN II with an $18 M_{\odot}$ progenitor as a function of distance to the source, using simulation results from [18]. The reference model refers to an explosion without axion production. The filled regions indicate the central $68 \%$ distribution of 200 realizations of each model. The dashed horizontal bands refer to the $3 \sigma$ and $5 \sigma$ thresholds.

Figure 3 shows the distribution of the detection significance $\xi$ of Galactic SNe II as a function of distance from Earth, assuming the reference model $g_{a p}=0$ and an axion production model $g_{a p} \neq 0$. In all cases a signal bin width of $10 \mathrm{~s}$ was used to compute the likelihood in eq. (2.1), and at each location 100 realizations of the SN II were generated to produce a $68 \%$ envelope for the significance distribution.

For any range of distances within $15 \mathrm{kpc}$ of Earth the detection of this SN II is highly significant. The two scenarios are also relatively well-separated in that axion cooling strongly affects the strength of the signal for nearby explosions. For $d>15 \mathrm{kpc}$, the two scenarios become increasingly indistinguishable from each other and from fluctuations in the detector background.

\subsection{Shape Analysis: Definition and Results}

To discriminate between the reference and axion scenarios, we compute a test statistic based on the time required for $90 \%$ of the excess counts above background to be observed in IceCube. This quantity, which we denote $t_{90}$, is sensitive to relative differences in the shape of the cooling 
tail shown in Fig. 2. Simulations indicate that $t_{90}$ is insensitive to systematic uncertainties in the normalization of the neutrino emission model of up to $25 \%$.

Given a realization of the $18 M_{\odot}$ SN II at varying distances from Earth, we can construct a distribution of $t_{90}$ for the scenario with no axion production $\left(H_{0}\right)$ and the scenario with axion production $\left(H_{a}\right)$. The resulting distributions are shown in Fig. 4 for two representative distances: $5 \mathrm{kpc}$ from Earth (left) and $10 \mathrm{kpc}$ from Earth (right). The distributions are normalized to provide an estimate of $p\left(t_{90} \mid H_{i}, d\right)$, the probability of observing a particular value of $t_{90}$ given the distance $d$ distance to the supernova and the model $H_{i}$ under consideration.
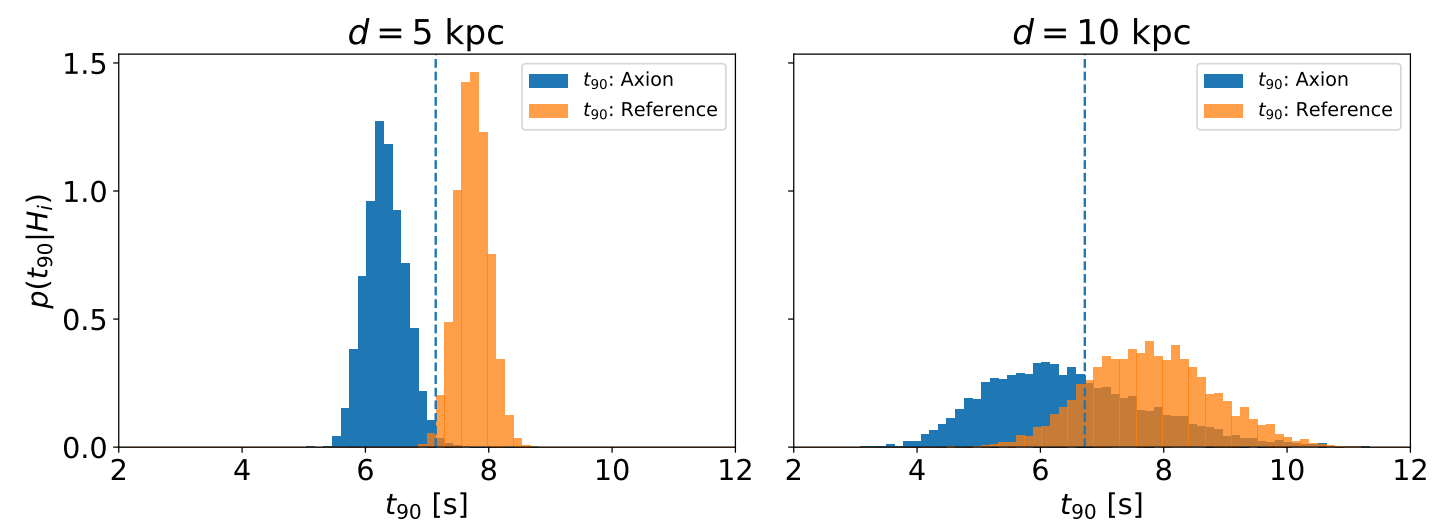

Figure 4: Distribution of $t_{90}$, the $90^{\text {th }}$ percentile of the cumulative DOM hit light curve after baseline subtraction, for SN II models with axion production and without ("reference"), for an explosion $5 \mathrm{kpc}$ and $10 \mathrm{kpc}$ from Earth. The dashed line indicates an optimal cut on $t_{90}$ that balances the false positive and false negative error obtained rates during model selection between the axion and reference models.

To discriminate between the two models, we construct a two-sided Neyman-Pearson hypothesis test that balances two quantities:

1. The Type I (or "false positive") error rate $\alpha$, defined as the tail probability of falsely rejecting the reference model $H_{0}$. From Fig. 4 ,

$$
\alpha=\int_{-\infty}^{t_{\mathrm{cut}}} p\left(t_{90} \mid H_{0}\right) d t_{90}
$$

2. The Type II (or "false negative") error rate, defined as the tail probability of falsely rejecting the axion model $H_{a}$. From Fig. 4 ,

$$
\beta=\int_{t_{\mathrm{cut}}}^{\infty} p\left(t_{90} \mid H_{a}\right) d t_{90}
$$

The value of $t_{\text {cut }}$ used as the decision point between $H_{0}$ and $H_{a}$ depends on the desired significance $\alpha$ and power $1-\beta$ of the test. In Fig. 4 we indicate possible values of $t_{\text {cut }}$ with a vertical dashed line. The particular choice shown in the figure balances the error rates $\alpha$ and $\beta$, but other choices are possible. For example, we may fix $t_{\text {cut }}$ such that the test provides $\alpha \leq 0.0027$ ( $3 \sigma$ ) or $\alpha \leq 6 \times 10^{-7}$ $(5 \sigma)$ evidence against $H_{0}$, and evaluate the resulting false negative rate $\beta$. 

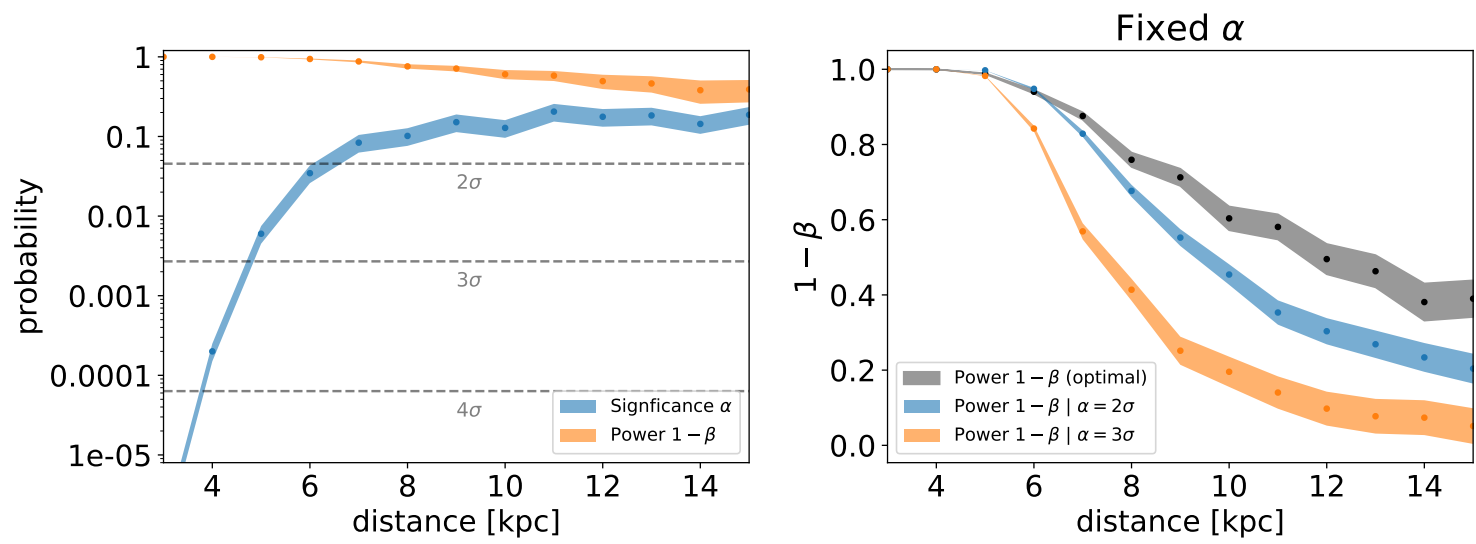

Figure 5: Left: dependence of the statistical significance $\alpha$ and statistical power $1-\beta$ of an hypothesis test between the axion and reference models as a function of distance to the supernova, assuming a test boundary that balances the false positive and false negative error rates. The filled bands indicate the statistical uncertainties of the simulation. Right: dependence of the statistical power of the hypothesis test for fixed values of $\alpha$. For reference, the top curve shows the statistical power for the "balanced" test boundary.

The statistical significance and power of the Neyman-Pearson test is shown in Figure 5 as a function of distance $d$ to the supernova remnant. In the left panel of the figure, $t_{\text {cut }}$ has been chosen to balance the false positive and false negative error rates $\alpha$ and $\beta$, as described in Fig. 4 . In the right panel $\alpha$ has been fixed to the $2 \sigma$ and $3 \sigma$ thresholds, and the resulting values of $t_{\text {cut }}$ and $1-\beta$ were computed.

The constructed test suggests that IceCube would not be able to easily identify the axion production scenario in a supernova occurring $8 \mathrm{kpc}$ away in the Galactic Center. In the case of the "balanced" test, simulations of an explosion at the Galactic Center produce $\alpha \approx 0.1$ and $1-\beta \approx 0.8$. In the case where $\alpha=0.0027(3 \sigma)$, the typical threshold used for evidence against the reference hypothesis, the statistical power of the test is $\sim 40 \%$; i.e., the production of axions is ruled out in the majority of realizations of the explosion.

However, the discrimination power of the test improves inversely with distance to the supernova. Simulations indicate that IceCube would exceed the $5 \sigma$ discovery threshold for axion productions with supernovae located at $d \leq 3 \mathrm{kpc}$ from Earth.

\section{Conclusions and Discussion}

The simulation of a Galactic Type II supernova with and without the production of axions in the proto-neutron star suggests that IceCube has substantial sensitivity to the deficit of neutrinos caused by axion cooling within a limited detection horizon. The two-sided hypothesis test described in Section 3 is robust in the presence of systematic uncertainties in the model flux, and indicates that IceCube can reach the $5 \sigma$ discovery threshold for SN II within $3 \mathrm{kpc}$ of Earth. For an SN II in the Galactic Center, IceCube would provide $3 \sigma$ evidence for axion production with moderate statistical power. Model discrimination becomes effectively impossible for distances beyond the Galactic Center. 
Given the low rate of Type II supernovae in the Milky Way, a large degree of luck is required for IceCube to detect axion production in a Galactic SN II using the test described above. Therefore, the possibility of improving the significance of this detection, as well as extending the detection horizon, will be the subject of future work.

\section{References}

[1] M. S. Turner, Phys. Rept. 197 (1990) 67-97.

[2] K. A. Olive et al., Chin. Phys. C38 (2014) 090001.

[3] A. Ringwald, Axions and Axion-Like Particles, in Proc. 49th Rencontres de Moriond, pp. 223-230, 2014. arXiv:1407.0546.

[4] R. D. Peccei and H. R. Quinn, Phys. Rev. Lett. 38 (1977) 1440-1443.

[5] G. Raffelt and L. Stodolsky, Phys. Rev. D37 (1988) 1237.

[6] E. Armengaud et al., JINST 9 (2014) T05002.

[7] D. Horns, L. Maccione, M. Meyer, A. Mirizzi, D. Montanino, and M. Roncadelli, Phys. Rev. D86 (2012) 075024.

[8] D. Hooper and P. D. Serpico, Phys. Rev. Lett. 99 (2007) 231102.

[9] H.E.S.S. Collaboration, A. Abramowski et al., Phys. Rev. D88 (2013) 102003.

[10] Fermi-LAT Collaboration, M. Ajello et al., Phys. Rev. Lett. 116 (2016) 161101.

[11] G. G. Raffelt and D. S. P. Dearborn, Phys. Rev. D36 (1987) 2211.

[12] A. Friedland, M. Giannotti, and M. Wise, Phys. Rev. Lett. 110 (2013) 061101.

[13] A. Ayala, I. Domınguez, M. Giannotti, A. Mirizzi, and O. Straniero, Phys. Rev. Lett. 113 (2014) 191302.

[14] J. W. Brockway, E. D. Carlson, and G. G. Raffelt, Phys. Lett. B383 (1996) 439-443.

[15] A. Payez, C. Evoli, T. Fischer, M. Giannotti, A. Mirizzi, and A. Ringwald, JCAP 1502 (2015) 006.

[16] M. Meyer, M. Giannotti, A. Mirizzi, J. Conrad, and M. Sánchez-Conde, Phys. Rev. Lett. 118 (2017) 011103.

[17] M. S. Turner, Phys. Rev. Lett. 60 (1988) 1797.

[18] T. Fischer, S. Chakraborty, M. Giannotti, A. Mirizzi, A. Payez, and A. Ringwald, Phys. Rev. D94 (2016) 085012.

[19] A. Payez, A fresh look on the limit on ultralight axion-like particles from SN1987A, in Proc. AXION-WIMP 2014: Geneva, Switzerland, pp. 121-124, 2014. arXiv:1410.4404.

[20] W. Keil, H.-T. Janka, D. N. Schramm, G. Sigl, M. S. Turner, and J. R. Ellis, Phys. Rev. D56 (1997) 2419-2432.

[21] IceCube Collaboration, R. Abbasi et al., Astron. Astrophys. 535 (2011) A109. [Erratum: Astron. Astrophys.563,C1(2014)].

[22] P. Antonioli et al., New J. Phys. 6 (2004) 114. 